\title{
APPROXIMATE CONTROLLABILITY OF IMPULSIVE NON-LOCAL NON-LINEAR FRACTIONAL DYNAMICAL SYSTEMS AND OPTIMAL CONTROL
}

\author{
SARRA GUECHI, AMAR DEBBOUCHE, AND DELFIM F. M. TORRES
}

Received 24 December, 2017

\begin{abstract}
We establish existence, approximate controllability and optimal control of a class of impulsive non-local non-linear fractional dynamical systems in Banach spaces. We use fractional calculus, sectorial operators and Krasnoselskii fixed point theorems for the main results. Approximate controllability results are discussed with respect to the inhomogeneous non-linear part. Moreover, we prove existence results of optimal triplets of corresponding fractional control systems with Bolza cost functionals.
\end{abstract}

2010 Mathematics Subject Classification: 26A33; 45J05; 49J15; 93B05

Keywords: fractional nonlinear equations, approximate controllability, $q$-resolvent families, optimal control, nonlocal and impulsive conditions

\section{INTRODUCTION}

We are concerned with an impulsive non-local non-linear fractional control dynamical system of form

$$
\left\{\begin{array}{l}
{ }^{C} D_{t}^{q} x(t)=A x(t)+f(t, x(t),(H x)(t))+B u(t), t \in(0, b] \backslash\left\{t_{1}, t_{2}, \ldots, t_{m}\right\}, \\
x(0)+g(x)=x_{0} \in X, \quad \triangle x\left(t_{i}\right)=I_{i}\left(x\left(t_{i}^{-}\right)\right)+D v\left(t_{i}^{-}\right), \quad i=1,2, \ldots, m,
\end{array}\right.
$$

where ${ }^{C} D_{t}^{q}$ is the Caputo fractional derivative of order $0<q<1$, the state $x(\cdot)$ takes its values in a Banach space $X$ with norm $\|\cdot\|$, and $x_{0} \in X$. Let $A: D(A) \subset X \rightarrow X$ be a sectorial operator of type $(M, \theta, q, \mu)$ on $X, H: I \times I \times X \rightarrow X$ represents a Volterra-type operator such that $(H x)(t)=\int_{0}^{t} h(t, s, x(s)) d s$, the control functions $u(\cdot)$ and $v(\cdot)$ are given in $L^{2}(I, U), U$ is a Banach space, $B$ and $D$ are bounded linear operators from $U$ into $X$. Here, one has $I=[0, b], 0=t_{0}<t_{1}<\cdots<t_{m}<t_{m+1}=b$, $I_{i}: X \rightarrow X$ are impulsive functions that characterize the jump of the solutions at impulse points $t_{i}$, the non-linear term $f: I \times X \times X \rightarrow X$, the non-local function $g: P C(I, X) \rightarrow X$, with $P C$ defined later, $\triangle x\left(t_{i}\right)=x\left(t_{i}^{+}\right)-x\left(t_{i}^{-}\right)$, where $x\left(t_{i}^{+}\right)$ and $x\left(t_{i}^{-}\right)$are the right and left limits of $x$ at the point $t_{i}$, respectively. 
Derivatives and integrals of arbitrary order, the main objects of Fractional Calculus (FC), have kept the interest of many scientists in recent years, since they provide an excellent tool to describe hereditary properties of various materials and processes. During the past decades, FC and its applications have gained a lot of importance, due to successful results in modelling several complex phenomena in numerous seemingly diverse and widespread fields of science and engineering, such as heat conduction, diffusion, propagation of waves, radiative transfer, kinetic theory of gases, diffraction problems and water waves, radiation, continuum mechanics, geophysics, electricity and magnetism, as well as in mathematical economics, communication theory, population genetics, queuing theory and medicine. For details on the theory and applications of FC see [9]. For recent developments in non-local and impulsive fractional differential problems see $[1,2,8,10]$ and references therein.

The problem of controllability is one of the most important qualitative aspects of dynamical systems in control theory. It consists to show the existence of a control function that steers the solution of the system from its initial state to a final state, where the initial and final states may vary over the entire space. This concept plays a major role in finite-dimensional control theory, so that it is natural to try to generalize it to infinite dimensions [14]. Moreover, exact controllability for semi-linear fractional order systems, when the non-linear term is independent of the control function, is proved by assuming that the controllability operator has an induced inverse on a quotient space. However, if the semi-group associated with the system is compact, then the controllability operator is also compact and hence the induced inverse does not exist because the state space is infinite dimensional [17]. Thus, the concept of exact controllability is too strong and has limited applicability, while approximate controllability is a weaker concept completely adequate in applications.

On the other hand, control systems are often based on the principle of feedback, where the signal to be controlled is compared to a desired reference, and the discrepancy is used to compute a corrective control action. Fractional optimal control of a distributed system is an optimal control problem for which the system dynamics is defined with fractional differential equations. Recently, attention has been paid to prove existence, approximate controllability and/or optimal control for different classes of fractional differential equations [4-7].

In [11], optimal control of non-instantaneous impulsive differential equations is studied. Qin et al. investigate approximate controllability and optimal control of fractional dynamical systems of order $1<q<2$ in Banach spaces [13]. Debbouche and Antonov established approximate controllability of semi-linear Hilfer fractional differential inclusions with impulsive control inclusion conditions in Banach spaces [3]. Motivated by the above works, here we construct an impulsive non-local nonlinear fractional control dynamical system and prove new sufficient conditions to treat the questions of approximate controllability and optimal control. 
The paper is organized as follows. In Section 2, we recall some facts from fractional calculus, $q$-resolvent families, and useful versions of fixed point techniques that are used for obtaining our main results. In Section 3, we form appropriate sufficient conditions and prove existence results for the fractional control system (1.1). In Section 4, we investigate the question of approximate controllability. We end with Section 5, where we obtain optimal controls corresponding to fractional control systems with a Bolza cost functional.

\section{PRELIMINARIES}

Here we present some preliminaries from fractional calculus [9], operator theory [12] and fixed point techniques [1], which are used throughout the work to obtain the desired results.

Definition 1. The left-sided Riemann-Liouville fractional integral of order $\alpha>0$, with lower limit $a$, for a function $f:[a,+\infty) \rightarrow \mathbb{R}$, is defined as

$$
I_{a^{+}}^{\alpha} f(t)=\frac{1}{\Gamma(\alpha)} \int_{a}^{t}(t-s)^{\alpha-1} f(s) d s
$$

provided the right side is point-wise defined on $[a,+\infty)$, where $\Gamma(\cdot)$ is the Euler gamma function. If $a=0$, then we can write $I_{0^{+}}^{\alpha} f(t)=\left(g_{\alpha} * f\right)(t)$, where

$$
g_{\alpha}(t):= \begin{cases}\frac{1}{\Gamma(\alpha)} t^{\alpha-1}, & t>0, \\ 0, & t \leq 0,\end{cases}
$$

and $*$ denotes convolution of functions. Moreover, $\lim _{\alpha \rightarrow 0} g_{\alpha}(t)=\delta(t)$, with $\delta$ the delta Dirac function.

Definition 2. The left-sided Riemann-Liouville fractional derivative of order $\alpha>$ $0, n-1 \leq \alpha<n, n \in \mathbb{N}$, for a function $f:[a,+\infty) \rightarrow \mathbb{R}$, is defined by

$$
{ }^{L} D_{a^{+}}^{\alpha} f(t)=\frac{1}{\Gamma(n-\alpha)} \frac{d^{n}}{d t^{n}} \int_{a}^{t} \frac{f(s)}{(t-s)^{\alpha+1-n}} d s, \quad t>a,
$$

where function $f$ has absolutely continuous derivatives up to order $n-1$.

Definition 3. The left-sided Caputo fractional derivative of order $\alpha>0, n-1<$ $\alpha<n, n \in \mathbb{N}$, for a function $f:[a,+\infty) \rightarrow \mathbb{R}$, is defined by

$$
{ }^{C} D_{a^{+}}^{\alpha} f(t)=\frac{1}{\Gamma(n-\alpha)} \int_{a}^{t} \frac{f^{(n)}(s)}{(t-s)^{\alpha+1-n}} d s=I_{a^{+}}^{n-\alpha} f^{(n)}(t), \quad t>a,
$$

where function $f$ has absolutely continuous derivatives up to order $n-1$.

Throughout the paper, by $P C(I, X)$ we denote the space of $X$-valued bounded functions on $I$ with the uniform norm $\|x\|_{P C}=\sup \{\|x(t)\|, t \in I\}$ such that $x\left(t_{i}^{+}\right)$ exists for any $i=0, \ldots, m$ and $x(t)$ is continuous on $\left(t_{i}, t_{i+1}\right], i=0, \ldots, m, t_{0}=0$ and $t_{m+1}=b$. 
Definition 4 (See [16]). Let $A: D \subseteq X \rightarrow X$ be a closed and linear operator. We say that $A$ is sectorial of type $(M, \theta, q, \mu)$, if there exists $\mu \in \mathbb{R}, 0<\theta<\frac{\pi}{2}$ and $M>0$ such that the $q$-resolvent of $A$ exists outside the sector

$$
\mu+S_{\theta}=\left\{\mu+\lambda^{q}: \lambda \in \mathbb{C},\left|\operatorname{Arg}\left(-\lambda^{q}\right)\right|<\theta\right\}
$$

and

$$
\left\|\left(\lambda^{q} I-A\right)^{-1}\right\| \leq \frac{M}{\left|\lambda^{q}-\mu\right|}, \lambda^{q} \notin \mu+S_{\theta} .
$$

Remark 1. If $A$ is a sectorial operator of type $(M, \theta, q, \mu)$, then it is not difficult to see that $A$ is the infinitesimal generator of a $q$-resolvent family $T_{q}(t)_{t \geq 0}$ in a Banach space, where $T_{q}(t)=\frac{1}{2 \pi i} \int_{c} e^{\lambda t} R\left(\lambda^{q}, A\right) d \lambda$.

Definition 5 (Motivated by [3,16]). A state function $x \in P C(I, X)$ is called a mild solution of (1.1) if it satisfies the following integral equations:

$$
x(t)=S_{q}(t)\left(x_{0}-g(x)\right)+\int_{0}^{t} T_{q}(t-s)(f(s, x(s),(H x)(s))+B u(s)) d s
$$

if $t \in\left[0, t_{1}\right]$, and

$$
\begin{aligned}
x(t)=S_{q}\left(t-t_{i}\right)\left[x\left(t_{i}^{-}\right)+I_{i}(\right. & \left.\left.x\left(t_{i}^{-}\right)\right)+D v\left(t_{i}^{-}\right)\right] \\
& +\int_{t_{i}}^{t} T_{q}(t-s)[f(s, x(s),(H x)(s))+B u(s)] d s
\end{aligned}
$$

if $t \in\left(t_{i}, t_{i+1}\right], i=1, \ldots, m$, where

$$
S_{q}(t)=\frac{1}{2 \pi i} \int_{c} e^{\lambda t} \lambda^{q-1} R\left(\lambda^{q}, A\right) d \lambda \quad \text { and } \quad T_{q}(t)=\frac{1}{2 \pi i} \int_{c} e^{\lambda t} R\left(\lambda^{q}, A\right) d \lambda
$$

with $c$ being a suitable path such that $\lambda^{q} \notin \mu+S_{\theta}$ for $\lambda \in c$.

Let $x_{t_{k}}\left(x(0), \triangle x\left(t_{k-1}\right) ; u, v\right), k=1, \ldots, m+1$, be the state value of (1.1) at time $t_{k}$, corresponding to the non-local initial value $x(0)$, the impulsive values $\Delta x\left(t_{k-1}\right)=$ $x\left(t_{k-1}^{+}\right)-x\left(t_{k-1}^{-}\right)$and the controls $u$ and $v$. For every $x(0)$ and $\triangle x\left(t_{k-1}\right) \in X$, we introduce the set

$$
\mathfrak{R}\left(t_{k}, x(0), \Delta x\left(t_{k-1}\right)\right)=\left\{x_{t_{k}}\left(x(0), \Delta x\left(t_{k-1}\right) ; u, v\right): u(\cdot), v(\cdot) \in L^{2}(I, U)\right\},
$$

which is called the reachable set of system (1.1) at time $t_{k}$ (if $k=m+1$, then $t_{k}$ is the terminal time). Its closure in $X$ is denoted by $\overline{\mathfrak{R}\left(t_{k}, x(0), \Delta x\left(t_{k-1}\right)\right)}$.

Definition 6. The impulsive control system (1.1) is said to be approximately controllable on $I$ if $\overline{\Re\left(t_{k}, x(0), \Delta x\left(t_{k-1}\right)\right)}=X$, that is, given an arbitrary $\epsilon>0$, it is possible to steer from the points $x(0)$ and $\Delta x\left(t_{k-1}\right)$ at time $t_{k}$ all points in the state space $X$ within a distance $\epsilon$. 
Consider the linear impulsive fractional control system

$$
\left\{\begin{array}{l}
{ }^{C} D_{t}^{q} x(t)=A x(t)+B u(t), \\
x(0)=x_{0} \in X, \\
\triangle x\left(t_{i}\right)=D v\left(t_{i}^{-}\right), \quad i=1, \ldots, m .
\end{array}\right.
$$

Approximate controllability for the linear impulsive control fractional system (2.1) is a natural generalization of the notion of approximate controllability of a linear firstorder control system $\left(q=1\right.$ and $t_{i}=D=0, i=1,2, \ldots, m$, i.e., $t \in\left[t_{m}, t_{m+1}\right]=$ $[0, b])$. The controllability operators associated with (2.1) are

$$
\begin{aligned}
& \Psi_{t_{k-1}, 1}^{t_{k}}=\int_{t_{k-1}}^{t_{k}} T_{q}\left(t_{k}-s\right) B B^{*} T_{q}^{*}\left(t_{k}-s\right) d s, \quad k=1, \ldots, m+1, \\
& \Psi_{t_{k-1}, 2}^{t_{k}}=S_{q}\left(t_{k}-t_{k-1}\right) D D^{*} S_{q}^{*}\left(t_{k}-t_{k-1}\right), \quad k=2, \ldots, m+1,
\end{aligned}
$$

where $T_{q}^{*}(\cdot), S_{q}^{*}(\cdot), B^{*}$ and $D^{*}$ denote the adjoints of $T_{q}(\cdot), S_{q}(\cdot), B$ and $D$, respectively. Moreover, for $\lambda>0$, we consider the relevant operator

$$
\mathcal{R}\left(\lambda, \Psi_{t_{k-1}, i}^{t_{k}}\right)=\left(\lambda I+\Psi_{t_{k-1}, i}^{t_{k}}\right)^{-1}, \quad i=1,2 .
$$

It is easy to verify that $\Psi_{t_{k-1}, 1}^{t_{k}}$ and $\Psi_{t_{k-1}, 2}^{t_{k}}$ are linear bounded operators.

Lemma 1 (See [3]). The linear impulsive control fractional system (2.1) is approximately controllable on I if and only if $\lambda \mathcal{R}\left(\lambda, \Psi_{t_{k-1}, i}^{t_{k}}\right) \rightarrow 0$ as $\lambda \rightarrow 0^{+}, i=1,2$, in the strong operator topology.

Lemma 2 (Krasnoselskii theorem [15]). Let $X$ be a Banach space and $E$ be a bounded, closed, and convex subset of $X$. Let $Q_{1}, Q_{2}$ be maps of $E$ into $X$ such that $Q_{1} x+Q_{2} y \in E$ for every $x, y \in E$. If $Q_{1}$ is a contraction and $Q_{2}$ is compact and continuous, then equation $Q_{1} x+Q_{2} x=x$ has a solution on $E$.

\section{EXISTENCE OF A MILD SOLUTION}

We prove existence for system (1.1). Define $K_{i}^{*}=\sup _{t \in I} \int_{t_{i-1}}^{t_{i}} m(t, s) d s<\infty$, $i=1, \ldots, m+1$. For any $r>0$, let $\Omega_{r}:=\{x \in P C(I, X) \mid\|x\| \leq r\}$. We make the following assumptions:

$\left(\mathrm{H}_{1}\right)$ The operators $S_{q}(t)_{t \geq 0}$ and $T_{q}(t)_{t \geq 0}$, generated by $A$, are bounded and compact, such that $\sup _{t \in I}\left\|S_{q}(t)\right\| \leq M$ and $\sup _{t \in I}\left\|T_{q}(t)\right\| \leq M$.

$\left(\mathrm{H}_{2}\right)$ The non-linearity $f: I \times X \times X \rightarrow X$ is continuous and compact; there exist functions $\mu_{i} \in L^{\infty}\left(I, \mathbb{R}^{+}\right), i=1,2,3$, and positive constants $\alpha_{1}$ and $\alpha_{2}$ such that $\|f(t, x, y)\| \leq \mu_{1}(t)+\mu_{2}(t)\|x\|+\mu_{3}(t)\|y\|$ and $\| f(t, x, H x)-$ $f(t, y, H y)\left\|=\alpha_{1}\right\| x-y\left\|+\alpha_{2}\right\| H x-H y \|$.

$\left(\mathrm{H}_{3}\right)$ Function $g: P C(I, X) \rightarrow X$ is completely continuous and there exists a positive constant $\beta$ such that $\|g(x)-g(y)\| \leq \beta\|x-y\|, x, y \in X$. 
$\left(\mathrm{H}_{4}\right)$ Associated with $h: \Delta \times X \rightarrow X$, there exists $m(t, s) \in P C\left(\Delta, \mathbb{R}^{+}\right)$such that $\|h(t, s, x(s))\| \leq m(t, s)\|x\|$ for each $(t, s) \in \Delta$ and $x, y \in X$, where $\Delta=$ $\left\{(t, s) \in \mathbb{R}^{2} \mid t_{i} \leq s, t \leq t_{i+1}, i=0, \ldots, m\right\}$.

$\left(\mathrm{H}_{5}\right)$ For every $x_{1}, x_{2}, x \in X$ and $t \in\left(t_{i}, t_{i+1}\right], i=1, \ldots, m, I_{i}$ are continuous and compact and there exist positive constants $d_{i}, e_{i}$ such that

$$
\begin{aligned}
& \qquad\left\|I_{i}\left(x_{1}\left(t_{i}^{-}\right)\right)-I_{i}\left(x_{2}\left(t_{i}^{-}\right)\right)\right\| \leq d_{i} \sup _{t \in\left(t_{i}, t_{i+1}\right]}\left\|x_{1}(t)-x_{2}(t)\right\| \\
& \text { and }\left\|I_{i}\left(x\left(t_{i}^{-}\right)\right)\right\| \leq e_{i} \sup _{t \in\left(t_{i}, t_{i+1}\right]}\|x(t)\| .
\end{aligned}
$$

Theorem 1. Let $x_{0} \in X$. If conditions $\left(H_{1}\right)-\left(H_{5}\right)$ hold, then the impulsive nonlocal fractional control system (1.1) has a fixed point on $I$ provided $M \beta<1$ and $M\left(1+d_{i}\right)<1, i=1, \ldots, m$, that is, (1.1) has at least one mild solution on $t \in$ $[0, b] \backslash\left\{t_{1}, \ldots, t_{m}\right\}$.

Proof. Define the operators $Q_{1}$ and $Q_{2}$ on $\Omega_{r}$ as follows:

$$
\begin{aligned}
& \left(Q_{1} x\right)(t)=\left\{\begin{array}{l}
S_{q}(t)\left(x_{0}-g(x)\right), \quad t \in\left[0, t_{1}\right] \\
S_{q}\left(t-t_{i}\right)\left[x\left(t_{i}^{-}\right)+I_{i}\left(x\left(t_{i}^{-}\right)\right)+D v\left(t_{i}^{-}\right)\right], \quad t \in\left(t_{i}, t_{i+1}\right],
\end{array}\right. \\
& \left(Q_{2} x\right)(t)= \begin{cases}\left.\int_{0}^{t} T_{q}(t-s)\right)(f(s, x(s),(H x)(s))+B u(s)) d s, & t \in\left[0, t_{1}\right], \\
\left.\int_{t_{i}}^{t} T_{q}(t-s)\right)(f(s, x(s),(H x)(s))+B u(s)) d s, & t \in\left(t_{i}, t_{i+1}\right],\end{cases}
\end{aligned}
$$

$i=1, \ldots, m$. We take the controls

$$
\begin{aligned}
& u=B^{*} T_{q}^{*}\left(t_{k}-t\right) \mathcal{R}\left(\lambda, \Psi_{t_{k-1}, 1}^{t_{k}}\right) P_{1}^{k}(x(\cdot)), \\
& v=D^{*} S_{q}^{*}\left(t_{k}-t_{k-1}\right) \mathcal{R}\left(\lambda, \Psi_{t_{k-1}, 2}^{t_{k}}\right) P_{2}^{k}(x(\cdot)),
\end{aligned}
$$

where

$$
\begin{aligned}
& P_{1}^{k}(x(\cdot))=\left\{\begin{array}{c}
x_{1}-S_{q}\left(t_{1}\right)\left(x_{0}-g(x)\right) \\
\quad-\int_{0}^{t_{1}} T_{q}\left(t_{1}-s\right) f(s, x(s),(H x)(s)) d s, \quad k=1, \\
x_{k}-S_{q}\left(t_{k}-t_{k-1}\right)\left[x\left(t_{k-1}^{-}\right)+I_{k-1}\left(x\left(t_{k-1}^{-}\right)\right)\right] \\
-\int_{t_{k-1}}^{t_{k}} T_{q}\left(t_{k}-s\right) f(s, x(s),(H x)(s)) d s, \quad k=2, \ldots, m+1,
\end{array}\right. \\
& P_{2}^{k}(x(\cdot))=\left\{\begin{array}{c}
x_{k}-S_{q}\left(t_{k}-t_{k-1}\right)\left[x\left(t_{k-1}^{-}\right)+I_{k-1}\left(x\left(t_{k-1}^{-}\right)\right)\right] \\
-\int_{t_{k-1}}^{t_{k}} T_{q}\left(t_{k}-s\right) f(s, x(s),(H x)(s)) d s, \quad k=2, \ldots, m+1 .
\end{array}\right.
\end{aligned}
$$

For any $\lambda>0$, we shall show that $Q_{1}+Q_{2}$ has a fixed point on $\Omega_{r}$, which is a solution of system (1.1). According to (3.1), together with (2.2) and (2.3), we have

$$
\|u(t)\| \leq \frac{1}{\lambda} M\|B\|\left\|P_{1}(x(\cdot))\right\| \text { and }\|v(t)\| \leq \frac{1}{\lambda} M\|D\|\left\|P_{2}(x(\cdot))\right\| .
$$


Using assumptions $\left(H_{1}\right)-\left(H_{5}\right)$, we get

$$
\begin{aligned}
\left\|P_{1}^{1}(x(\cdot))\right\| \leq & \left\|x_{1}\right\|+\left\|S_{q}\left(t_{1}\right)\right\|\left\|\left(x_{0}-g(x)\right)\right\| \\
& +\int_{0}^{t_{1}}\left\|T_{q}\left(t_{1}-s\right)\right\|\|f(s, x(s),(H x)(s))\| d s \\
\leq & \left\|x_{1}\right\|+M\left(\left\|x_{0}\right\|+\|g(x)\|\right) \\
& +M t_{1}\left(\left\|\mu_{1}\right\|_{L^{\infty}\left(I, \mathbb{R}^{+}\right)}+r\left\|\mu_{2}\right\|_{L^{\infty}\left(I, \mathbb{R}^{+}\right)}+K_{1}^{*} r\left\|\mu_{3}\right\|_{L^{\infty}\left(I, \mathbb{R}^{+}\right)}\right) \\
\leq & \left\|x_{1}\right\|+M\left\|x_{0}\right\|+M \beta\|x\|+M\|g(0)\| \\
& +M t_{1}\left(\left\|\mu_{1}\right\|_{L^{\infty}\left(I, \mathbb{R}^{+}\right)}+r\left\|\mu_{2}\right\|_{L^{\infty}\left(I, \mathbb{R}^{+}\right)}+K_{1}^{*} r\left\|\mu_{3}\right\|_{L^{\infty}\left(I, \mathbb{R}^{+}\right)}\right)
\end{aligned}
$$

and, for $k=2, \ldots, m+1$,

$$
\begin{aligned}
\left\|P_{1}^{k}(x(\cdot))\right\| \leq & \left\|x_{k}\right\|+\left\|S_{q}\left(t_{k}-t_{k-1}\right)\right\|\left[\left\|x\left(t_{k-1}^{-}\right)\right\|+\left\|I_{k-1}\left(x\left(t_{k-1}^{-}\right)\right)\right\|\right] \\
& +\int_{t_{k-1}}^{t_{k}}\left\|T_{q}\left(t_{k}-s\right)\right\|\|f(s, x(s),(H x)(s))\| d s \\
\leq & \left\|x_{k}\right\|+M\left(\left\|x\left(t_{k-1}^{-}\right)\right\|+e_{i}\|x\|\right)+M\left(t_{k}-t_{k-1}\right) \\
& \times\left(\left\|\mu_{1}\right\|_{L^{\infty}\left(I, \mathbb{R}^{+}\right)}+r\left\|\mu_{2}\right\|_{L^{\infty}\left(I, \mathbb{R}^{+}\right)}+K_{k}^{*} r\left\|_{3}\right\|_{L^{\infty}\left(I, \mathbb{R}^{+}\right)}\right) \\
\leq & \left\|x_{k}\right\|+M\left(\left\|x\left(t_{k-1}^{-}\right)\right\|+r e_{i}\right)+M\left(t_{k}-t_{k-1}\right) \\
& \times\left(\left\|\mu_{1}\right\|_{L^{\infty}\left(I, \mathbb{R}^{+}\right)}+r\left\|\mu_{2}\right\|_{L^{\infty}\left(I, \mathbb{R}^{+}\right)}+K_{k}^{*} r\left\|_{3}\right\|_{L^{\infty}\left(I, \mathbb{R}^{+}\right)}\right) .
\end{aligned}
$$

Similarly, we get

$$
\begin{aligned}
& \left\|P_{2}^{k}(x(\cdot))\right\| \leq\left\|x_{k}\right\|+M\left(\left\|x\left(t_{k-1}^{-}\right)\right\|+r e_{k-1}\right) \\
& \quad+M\left(t_{k}-t_{k-1}\right)\left(\left\|\mu_{1}\right\|_{L^{\infty}\left(I, \mathbb{R}^{+}\right)}+r\left\|\mu_{2}\right\|_{L^{\infty}\left(I, \mathbb{R}^{+}\right)}+K_{k}^{*} r\left\|\mu_{3}\right\|_{L^{\infty}\left(I, \mathbb{R}^{+}\right)}\right),
\end{aligned}
$$

$k=2, \ldots, m+1$. For any $x \in \Omega_{r}$, we obtain

$$
\begin{gathered}
\\
\left\|\left(Q_{1} x\right)(t)+\left(Q_{2} x\right)(t)\right\| \\
\leq M\left(\left\|x_{0}\right\|+\|g(x)\|\right) \\
+M t_{1}\left(\left\|\mu_{1}\right\|_{L^{\infty}\left(I, \mathbb{R}^{+}\right)}+r\left\|\mu_{2}\right\|_{L^{\infty}\left(I, \mathbb{R}^{+}\right)}+K_{1}^{*} r\left\|\mu_{3}\right\|_{L^{\infty}\left(I, \mathbb{R}^{+}\right)}+\|B\|\|u\|\right) \\
\leq M\left(\left\|x_{0}\right\|+\beta r+\|g(0)\|\right) \\
+M t_{1}\left(\left\|\mu_{1}\right\|_{L^{\infty}\left(I, \mathbb{R}^{+}\right)}+r\left\|\mu_{2}\right\|_{L^{\infty}\left(I, \mathbb{R}^{+}\right)}+K_{1}^{*} r\left\|\mu_{3}\right\|_{L^{\infty}\left(I, \mathbb{R}^{+}\right)}+\|B\|\|u\|\right)
\end{gathered}
$$

for $t \in\left[0, t_{1}\right]$, and

$$
\begin{aligned}
&\left\|\left(Q_{1} x\right)(t)+\left(Q_{2} x\right)(t)\right\| \\
& \leq M\left(\left\|x\left(t_{k-1}^{-}\right)\right\|+e_{k-1}\|x\|+\|D\|\left\|v\left(t_{k-1}^{-}\right)\right\|\right)+M\left(t_{k}-t_{k-1}\right) \\
& \times\left(\left\|\mu_{1}\right\|_{L^{\infty}\left(I, \mathbb{R}^{+}\right)}+r\left\|\mu_{2}\right\|_{L^{\infty}\left(I, \mathbb{R}^{+}\right)}+K_{k}^{*} r\left\|\mu_{3}\right\|_{L^{\infty}\left(I, \mathbb{R}^{+}\right)}+\|B\|\|u\|\right) \\
& \leq M\left(\left\|x\left(t_{k-1}^{-}\right)\right\|+e_{k-1} r+\|D\|\left\|v\left(t_{k-1}^{-}\right)\right\|+M\left(t_{k}-t_{k-1}\right)\right. \\
& \times\left(\left\|\mu_{1}\right\|_{L^{\infty}\left(I, \mathbb{R}^{+}\right)}+r\left\|\mu_{2}\right\|_{L^{\infty}\left(I, \mathbb{R}^{+}\right)}+K_{k}^{*} r\left\|\mu_{3}\right\|_{L^{\infty}\left(I, \mathbb{R}^{+}\right)}+\|B\|\|u\|\right)
\end{aligned}
$$


for $t \in\left(t_{k-1}, t_{k}\right]$. By the inequalities (3.2), we can find $\xi_{1}, \xi_{2}>0$ such that

$$
\left\|\left(Q_{1} x\right)(t)+\left(Q_{2} x\right)(t)\right\| \leq\left\{\begin{array}{l}
\xi_{1}, t \in\left[0, t_{1}\right], \\
\xi_{2}, t \in\left(t_{k-1}, t_{k}\right], k=2, \ldots, m+1 .
\end{array}\right.
$$

Hence, $Q_{1} x+Q_{2} x$ is bounded. Now, let $x, y \in \Omega_{r}$. We have

$$
\left\|\left(Q_{1} x\right)(t)-\left(Q_{1} y\right)(t)\right\| \leq\left\|S_{q}(t)\right\|\|g(x)-g(y)\| \leq M \beta\|x-y\|
$$

for $t \in\left[0, t_{1}\right]$ and

$$
\begin{aligned}
& \left\|\left(Q_{1} x\right)(t)-\left(Q_{1} y\right)(t)\right\| \\
& \quad \leq\left\|S_{q}\left(t-t_{k-1}\right)\right\|\left[\left\|x\left(t_{k-1}^{-}\right)-y\left(t_{k-1}^{-}\right)\right\|+\left\|I_{k-1}\left(x\left(t_{k-1}^{-}\right)\right)-I_{k-1}\left(y\left(t_{k-1}^{-}\right)\right)\right\|\right] \\
& \quad \leq M\left[\left\|x\left(t_{k-1}^{-}\right)-y\left(t_{k-1}^{-}\right)\right\|+d_{k-1}\|x-y\|\right]
\end{aligned}
$$

for $t \in\left(t_{k-1}, t_{k}\right], k=2, \ldots, m+1$. Since $M \beta<1$ and $M\left(1+d_{k-1}\right)<1, k=$ $2, \ldots, m+1$, it follows that $Q_{1}$ is a contraction mapping. Let $\left\{x_{n}\right\}$ be a sequence in $\Omega_{r}$ such that $x_{n} \rightarrow x \in \Omega_{r}$. Since $f$ and $g$ are continuous, i.e., for all $\epsilon>0$, there exists a positive integer $n_{0}$, such that for $n>n_{0} \| f\left(s, x_{n}(s),\left(H x_{n}\right)(s)\right)-$ $f(s, x(s),(H x)(s)) \| \leq \epsilon$ and $\left\|g\left(x_{n}\right)-g(x)\right\| \leq \epsilon$, the continuity of $I_{i}(x)$ on $\left(t_{i}, t_{i+1}\right]$ gives $\left\|I_{i}\left(x_{n}\left(t_{i}^{-}\right)\right)-I_{i}\left(x\left(t_{i}^{-}\right)\right)\right\| \leq \epsilon, i=1, \ldots, m$. Now, for all $t \in\left[0, t_{1}\right]$,

$$
\begin{aligned}
& \left\|\left(Q_{2} x_{n}\right)(t)-\left(Q_{2} x\right)(t)\right\| \\
& \leq \int_{0}^{t_{1}}\left\|T_{q}(t-\tau)\right\|\left\|B B^{*} T_{q}^{*}\left(t_{1}-\tau\right) \mathcal{R}\left(\lambda, \Psi_{t_{0}, 1}^{t_{1}}\right)\right\|\left[\left\|S_{q}\left(t_{1}\right)\left(g\left(x_{n}\right)-g(x)\right)\right\|\right. \\
& \left.+\int_{0}^{t_{1}}\left\|T_{q}\left(t_{1}-s\right)\right\| f\left(s, x_{n}(s),\left(H x_{n}\right)(s)\right)-f(s, x(s),(H x)(s)) \| d s\right] d \tau \\
& +\int_{0}^{t_{1}}\left\|T_{q}(t-s)\right\|\left\|f\left(s, x_{n}(s),\left(H x_{n}\right)(s)\right)-f(s, x(s),(H x)(s))\right\| d s \\
& \leq \frac{\epsilon}{\lambda} M^{3}\|B\|^{2} t_{1}\left(2 t_{1}+1\right) .
\end{aligned}
$$

Moreover, for all $t \in\left(t_{i}, t_{i+1}\right], i=1, \ldots, m$, one has

$$
\begin{aligned}
& \left\|\left(Q_{2} x_{n}\right)(t)-\left(Q_{2} x\right)(t)\right\| \\
& \leq \int_{t_{i}}^{t}\left\|T_{q}(t-\tau)\right\|\left\|B B^{*} T_{q}^{*}\left(t_{i+1}-\tau\right) \mathcal{R}\left(\lambda, \Psi_{t_{i}, 1}^{t_{i+1}}\right)\right\| \\
& \quad \times\left[\left\|S_{q}\left(t_{i+1}-t_{i}\right)\left[x_{n}\left(t_{i}^{-}\right)-x\left(t_{i}^{-}\right)+I_{i}\left(x_{n}\left(t_{i}^{-}\right)\right)-I_{i}\left(x\left(t_{i}^{-}\right)\right)\right]\right\|\right. \\
& \left.\left.\quad+\int_{t_{i}}^{t_{i+1}}\left\|T_{q}\left(t_{i+1}-s\right)\right\| f\left(s, x_{n}(s),\left(H x_{n}\right)(s)\right)-f(s, x(s),(H x)(s)) \| d s\right)\right] d \tau \\
& \quad+\int_{t_{i}}^{t}\left\|T_{q}(t-s)\right\| \|\left(f\left(s, x_{n}(s),\left(H x_{n}\right)(s)\right)-f(s, x(s),(H x)(s)) \| d s\right.
\end{aligned}
$$




$$
\leq \frac{2 \epsilon}{\lambda} M^{3}\|B\|^{2}\left(t_{i+1}-t_{i}\right)\left(t_{i+1}-t_{i}+1\right) .
$$

Therefore, $Q_{2}$ is continuous. Next, we prove the compactness of $Q_{2}$. For that, we first show that the set $\left\{\left(Q_{2} x\right)(t): x \in \Omega_{r}\right\}$ is relatively compact in $P C(I, X)$. By the assumptions of our theorem, we have

$$
\begin{gathered}
\left\|\left(Q_{2} x\right)(t)\right\| \\
\leq M t_{1}\left(\left\|\mu_{1}\right\|_{L^{\infty}\left(I, \mathbb{R}^{+}\right)}+r\left\|\mu_{2}\right\|_{L^{\infty}\left(I, \mathbb{R}^{+}\right)}+K_{1}^{*} r\left\|\mu_{3}\right\|_{L^{\infty}\left(I, \mathbb{R}^{+}\right)}+\|B\|\|u\|\right),
\end{gathered}
$$

for $t \in\left[0, t_{1}\right]$, and

$$
\begin{aligned}
& \left\|\left(Q_{2} x\right)(t)\right\| \leq M\left(t_{k}-t_{k-1}\right) \\
& \quad \times\left(\left\|\mu_{1}\right\|_{L^{\infty}\left(I, \mathbb{R}^{+}\right)}+r\left\|\mu_{2}\right\|_{L^{\infty}\left(I, \mathbb{R}^{+}\right)}+K_{k}^{*} r\left\|\mu_{3}\right\|_{L^{\infty}\left(I, \mathbb{R}^{+}\right)}+\|B\|\|u\|\right),
\end{aligned}
$$

for $t \in\left(t_{k-1}, t_{k}\right]$, which gives the uniformly boundedness of $\left\{\left(Q_{2} x\right)(t): x \in \Omega_{r}\right\}$. We now show that $Q_{2}\left(\Omega_{r}\right)$ is equicontinuous. Functions $\left\{\left(Q_{2} x\right)(t): x \in \Omega_{r}\right\}$ are equicontinuous at $t=0$. For any $x \in \Omega_{r}$, if $0<r_{1}<r_{2} \leq t_{1}$, then

$$
\begin{aligned}
\|\left(Q_{2} x\right) & \left(r_{2}\right)-\left(Q_{2} x\right)\left(r_{1}\right) \| \\
\leq & \int_{0}^{r_{1}}\left\|T_{q}\left(r_{2}-s\right)-T_{q}\left(r_{1}-s\right)\right\|[\|B u(s)\|+\|f(s, x(s),(H x)(s))\|] d s \\
& +\int_{r_{1}}^{r_{2}}\left\|T_{q}\left(r_{2}-s\right)\right\|[\|B u(s)\|+\|f(s, x(s),(H x)(s))\|] d s \\
\leq & {\left[r_{1}\left\|T_{q}\left(r_{2}-s\right)-T_{q}\left(r_{1}-s\right)\right\|+M\left(r_{2}-r_{1}\right)\right] } \\
& \times\left(\|B\|\|u\|+\left\|\mu_{1}\right\|_{L^{\infty}\left(I, \mathbb{R}^{+}\right)}+r\left\|\mu_{2}\right\|_{L^{\infty}\left(I, \mathbb{R}^{+}\right)}+K_{1}^{*} r\left\|\mu_{3}\right\|_{L^{\infty}\left(I, \mathbb{R}^{+}\right)}\right) .
\end{aligned}
$$

Similarly, if $t_{i}<r_{1}<r_{2} \leq t_{i+1}$, then

$$
\begin{aligned}
&\left\|\left(Q_{2} x\right)\left(r_{2}\right)-\left(Q_{2} x\right)\left(r_{1}\right)\right\| \\
& \leq \int_{t_{i}}^{r_{1}}\left\|T_{q}\left(r_{2}-s\right)-T_{q}\left(r_{1}-s\right)\right\|[\|B u(s)\|+\|f(s, x(s),(H x)(s))\|] d s \\
&+\int_{r_{1}}^{r_{2}}\left\|T_{q}\left(r_{2}-s\right)\right\|[\|B u(s)\|+\|f(s, x(s),(H x)(s))\|] d s \\
& \leq {\left[\left(r_{1}-t_{i}\right)\left\|T_{q}\left(r_{2}-s\right)-T_{q}\left(r_{1}-s\right)\right\|+M\left(r_{2}-r_{1}\right)\right] } \\
& \times\left(\|B\|\|u\|+\left\|\mu_{1}\right\|_{L^{\infty}\left(I, \mathbb{R}^{+}\right)}+r\left\|\mu_{2}\right\|_{L^{\infty}\left(I, \mathbb{R}^{+}\right)}+K_{i+1}^{*} r\left\|\mu_{3}\right\|_{L^{\infty}\left(I, \mathbb{R}^{+}\right)}\right) .
\end{aligned}
$$

From $\left(H_{1}\right)$, it follows the continuity of operator $T_{q}(\cdot)$ in the uniform operator topology. Thus, the right hand side of the above inequality tends to zero as $r_{2} \rightarrow r_{1}$. Therefore, $\left\{\left(Q_{2} x\right)(t): x \in \Omega_{r}\right\}$ is a family of equicontinuous functions. According to the infinite dimensional version of the Ascoli-Arzela theorem, it remains to prove that, for any $t \in[0, b] \backslash\left\{t_{1}, \ldots, t_{m}\right\}$, the set $V(t):=\left\{\left(Q_{2} x\right)(t): x \in \Omega_{r}\right\}$ is relatively compact in $P C(I, X)$. The case $t=0$ is trivial: $V(0)=\left\{\left(Q_{2} x\right)(0): x(\cdot) \in \Omega_{r}\right\}$ is 
compact in $P C(I, X)$. Let $t \in\left(0, t_{1}\right]$ be a fixed real number and $h$ be a given real number satisfying $0<h<t_{1}$. Define $V_{h}(t)=\left\{\left(Q_{2}^{h} x\right)(t): x \in \Omega_{r}\right\}$,

$$
\begin{aligned}
\left(Q_{2}^{h} x\right)(t)= & \int_{0}^{t-h} T_{q}(t-s) B u(s) d s+\int_{0}^{t-h} T_{q}(t-s) f(s, x(s),(H x)(s)) d s \\
= & T_{q}(h) \int_{0}^{t-h} T_{q}(t-s-h) B u(s) d s \\
& +T_{q}(h) \int_{0}^{t-h} T_{q}(t-s-h) f(s, x(s),(H x)(s)) d s \\
= & T_{q}(h) y_{1}(t, h) .
\end{aligned}
$$

We use same arguments, we fix $t \in\left(t_{i}, t_{i+1}\right]$, and let $h$ be a given real number satisfying $t_{i}<h<t_{i+1}$, we define $V_{h}(t)=\left\{\left(Q_{2}^{h} x\right)(t): x \in \Omega_{r}\right\}$,

$$
\begin{aligned}
\left(Q_{2}^{h} x\right)(t) & =\int_{t_{i}}^{t-h} T_{q}(t-s) B u(s) d s+\int_{t_{i}}^{t-h} T_{q}(t-s) f(s, x(s),(H x)(s)) d s \\
& =T_{q}(h) \int_{t_{i}}^{t-h} T_{q}(t-s-h) B u(s) d s \\
& +T_{q}(h) \int_{t_{i}}^{t-h} T_{q}(t-s-h) f(s, x(s),(H x)(s)) d s \\
& =T_{q}(h) y_{2}(t, h) .
\end{aligned}
$$

The compactness of $T_{q}(h)$ in $P C(I, X)$, together with the boundedness of both $y_{1}(t, h)$ and $y_{2}(t, h)$ on $\Omega_{r}$, give the relativity compactness of the set $V_{h}(t)$ in $P C(I, X)$. Moreover, for all $t \in\left[0, t_{1}\right]$,

$$
\begin{aligned}
& \left\|\left(Q_{2} x\right)(t)-\left(Q_{2}^{h} x\right)(t)\right\| \\
& \quad \leq \int_{t-h}^{t} T_{q}(t-s) B u(s) d s+\int_{t-h}^{t} T_{q}(t-s) f(s, x(s),(H x)(s)) d s \\
& \quad \leq h M\left(\|B\|\|u\|+\left\|\mu_{1}\right\|_{L^{\infty}\left(I, \mathbb{R}^{+}\right)}+r\left\|\mu_{2}\right\|_{L^{\infty}\left(I, \mathbb{R}^{+}\right)}+K_{1}^{*} r\left\|\mu_{3}\right\|_{L^{\infty}\left(I, \mathbb{R}^{+}\right)}\right) .
\end{aligned}
$$

Also, for all $t \in\left(t_{i}, t_{i+1}\right]$,

$$
\begin{aligned}
& \left\|\left(Q_{2} x\right)(t)-\left(Q_{2}^{h} x\right)(t)\right\| \\
& \quad \leq \int_{t-h}^{t} T_{q}(t-s) B u(s) d s+\int_{t-h}^{t} T_{q}(t-s) f(s, x(s),(H x)(s)) d s \\
& \quad \leq h M\left(\|B\|\|u\|+\left\|\mu_{1}\right\|_{L^{\infty}\left(I, \mathbb{R}^{+}\right)}+r\left\|\mu_{2}\right\|_{L^{\infty}\left(I, \mathbb{R}^{+}\right)}+K_{i+1}^{*} r\left\|\mu_{3}\right\|_{L^{\infty}\left(I, \mathbb{R}^{+}\right)}\right) .
\end{aligned}
$$

Choose $h$ small enough. It implies that there are relatively compact sets arbitrarily close to the set $V(t)$ for each $t \in[0, b] \backslash\left\{t_{1}, \ldots, t_{m}\right\}$. Then, $V(t), t \in[0, b] \backslash$ $\left\{t_{1}, \ldots, t_{m}\right\}$, is relatively compact in $P C(I, X)$. Since it is compact at $t=0$, we 
have the relatively compactness of $V(t)$ in $P C(I, X)$ for all $t \in[0, b] \backslash\left\{t_{1}, \ldots, t_{m}\right\}$. Hence, by the Arzela-Ascoli theorem, we conclude that $Q_{2}$ is compact. From Lemma 2, we ensure that the control system (1.1) has at least one mild solution on $t \in[0, b] \backslash\left\{t_{1}, \ldots, t_{m}\right\}$.

\section{Approximate controllability}

In this section, with help of the obtained existence theorem of mild solutions, we show an approximate controllability result for system (1.1).

Theorem 2. If $\left(H_{1}\right)-\left(H_{5}\right)$ are satisfied and $\lambda \mathcal{R}\left(\lambda, \Psi_{t_{k-1}, i}^{t_{k}}\right) \rightarrow 0$ in the strong operator topology as $\lambda \rightarrow 0^{+}, i=1,2$, then the impulsive non-local fractional control system (1.1) is approximately controllable on $t \in[0, b] \backslash\left\{t_{1}, \ldots, t_{m}\right\}$.

Proof. According to Theorem 1, $Q_{1}^{\lambda}+Q_{2}^{\lambda}$ has a fixed point in $\Omega_{r}$ for any $\lambda>0$. This implies that there exists $\bar{x}^{\lambda} \in\left(Q_{1}^{\lambda}+Q_{2}^{\lambda}\right)\left(\bar{x}^{\lambda}\right)$ such that

$$
\bar{x}^{\lambda}(t)=\left\{\begin{array}{l}
S_{q}(t)\left(x_{0}-\bar{g}^{\lambda}\left(\bar{x}^{\lambda}\right)\right) \\
\quad+\int_{0}^{t} T_{q}(t-s)\left[\bar{f}^{\lambda}\left(s, \bar{x}^{\lambda}(s),\left(H \bar{x}^{\lambda}\right)(s)\right)+B \bar{u}^{\lambda}(s)\right] d s, t \in\left[0, t_{1}\right], \\
S_{q}\left(t-t_{k-1}\right)\left[\bar{x}^{\lambda}\left(t_{k-1}^{-}\right)+\bar{I}_{k-1} \lambda\left(\bar{x}^{\lambda}\left(t_{k-1}^{-}\right)\right)+D \bar{v}^{\lambda}\left(t_{k-1}^{-}\right)\right] \\
\quad+\int_{t_{k-1}}^{t} T_{q}(t-s)\left[\bar{f} \lambda\left(s, \bar{x}^{\lambda}(s),\left(H \bar{x}^{\lambda}\right)(s)\right)+B \bar{u}^{\lambda}(s)\right] d s, t \in\left(t_{k-1}, t_{k}\right],
\end{array}\right.
$$

where for $t \in\left[0, t_{1}\right]$ we have

$$
\begin{aligned}
\bar{u}^{\lambda}=B^{*} T_{q}^{*}\left(t_{1}-t\right) \mathcal{R}\left(\lambda, \Psi_{0,1}^{t_{1}}\right)[ & x_{1}-S_{q}\left(t_{1}\right)\left(x_{0}-\bar{g}^{\lambda}\left(\bar{x}^{\lambda}\right)\right) \\
& \left.\quad-\int_{0}^{t_{1}} T_{q}\left(t_{1}-s\right) \bar{f}^{\lambda}\left(s, \bar{x}^{\lambda}(s),\left(H \bar{x}^{\lambda}\right)(s)\right) d s\right]
\end{aligned}
$$

while for $k=2, \ldots, m+1$

$$
\begin{aligned}
\bar{u}^{\lambda} & B^{*} T_{q}^{*}\left(t_{k}-t\right) \mathcal{R}\left(\lambda, \Psi_{t_{k-1}, 1}^{t_{k}}\right)[ \\
& x_{k}-S_{q}\left(t_{k}-t_{k-1}\right)\left[\bar{x}^{\lambda}\left(t_{k-1}^{-}\right)+{\overline{I_{k-1}}}^{\lambda}\left(\bar{x}^{\lambda}\left(t_{k-1}^{-}\right)\right)\right] \\
& \left.\quad-\int_{t_{k-1}}^{t_{k}} T_{q}\left(t_{k}-s\right) \bar{f}^{\lambda}\left(s, \bar{x}^{\lambda}(s),\left(H \bar{x}^{\lambda}\right)(s)\right) d s\right]
\end{aligned}
$$

and

$$
\begin{aligned}
\bar{v}^{\lambda}=D^{*} S_{q}^{*} & \left(t_{k}-t_{k-1}\right) \mathcal{R}\left(\lambda, \Psi_{t_{k-1}, 2}^{t_{k}}\right) \\
\times & {\left[x_{k}-S_{q}\left(t_{k}-t_{k-1}\right)\left[\bar{x}^{\lambda}\left(t_{k-1}^{-}\right)+\bar{I}_{k-1} \lambda\left(\bar{x}^{\lambda}\left(t_{k-1}^{-}\right)\right)\right]\right.}
\end{aligned}
$$




$$
\left.-\int_{t_{k-1}}^{t_{k}} T_{q}\left(t_{k}-s\right) \bar{f}^{\lambda}\left(s, \bar{x}^{\lambda}(s),\left(H \bar{x}^{\lambda}\right)(s)\right) d s\right] .
$$

Furthermore,

$$
\begin{aligned}
\bar{x}^{\lambda}\left(t_{1}\right)=S_{q}\left(t_{1}\right)\left(x_{0}-\bar{g}^{\lambda}\left(\bar{x}^{\lambda}\right)\right) & \\
+ & \int_{0}^{t_{1}} T_{q}\left(t_{1}-s\right)\left[\bar{f}^{\lambda}\left(s, \bar{x}^{\lambda}(s),\left(H \bar{x}^{\lambda}\right)(s)\right)+B \bar{u}^{\lambda}(s)\right] d s, \\
\bar{x}^{\lambda}\left(t_{k}\right)=S_{q}\left(t_{k}-t_{k-1}\right) & {\left[\bar{x}^{\lambda}\left(t_{k-1}^{-}\right)+\bar{I}_{k-1} \lambda\left(\bar{x}^{\lambda}\left(t_{k-1}^{-}\right)\right)+D \bar{v}^{\lambda}\left(t_{k-1}^{-}\right)\right] } \\
+ & \int_{t_{k-1}}^{t_{k}} T_{q}\left(t_{k}-s\right)\left[\bar{f}^{\lambda}\left(s, \bar{x}^{\lambda}(s),\left(H \bar{x}^{\lambda}\right)(s)\right)+B \bar{u}^{\lambda}(s)\right] d s,
\end{aligned}
$$

$k=2, \ldots, m+1$, with

$$
\begin{gathered}
x_{t_{1}-} \bar{x}^{\lambda}\left(t_{1}\right)=x_{1}-\Psi_{0,1}^{t_{1}} \mathcal{R}\left(\lambda, \Psi_{0,1}^{t_{1}}\right)\left\{x_{1}-S_{q}\left(t_{1}\right)\left(x_{0}-\bar{g}^{\lambda}\left(\bar{x}^{\lambda}\right)\right)\right. \\
\left.\quad-\int_{0}^{t_{1}} T_{q}\left(t_{1}-s\right) \bar{f}^{\lambda}\left(s, \bar{x}^{\lambda}(s),\left(H \bar{x}^{\lambda}\right)(s)\right) d s\right\} \\
-S_{q}\left(t_{1}\right)\left(x_{0}-\bar{g}^{\lambda}\left(\bar{x}^{\lambda}\right)\right)-\int_{0}^{t_{1}} T_{q}\left(t_{1}-s\right) \bar{f}^{\lambda}\left(s, \bar{x}^{\lambda}(s),\left(H \bar{x}^{\lambda}\right)(s)\right) d s, \\
x_{t_{k}}-\bar{x}^{\lambda}\left(t_{k}\right)=x_{k}-\Psi_{k-1,2}^{t_{k}} \mathcal{R}\left(\lambda, \Psi_{k-1,2}^{t_{k}}\right) \\
\times\left\{x_{k}-S_{q}\left(t_{k}-t_{k-1}\right)\left[\bar{x}^{\lambda}\left(t_{k-1}^{-}\right)+\bar{I}_{k-1} \lambda\left(\bar{x}^{\lambda}\left(t_{k-1}^{-}\right)\right)\right]\right. \\
\left.-\int_{t_{k-1}}^{t_{k}} T_{q}\left(t_{k}-s\right) \bar{f}^{\lambda}\left(s, \bar{x}^{\lambda}(s),\left(H \bar{x}^{\lambda}\right)(s)\right) d s\right\} \\
-S_{q}\left(t_{k}-t_{k-1}\right)\left[\bar{x}^{\lambda}\left(t_{k-1}^{-}\right)+\bar{I}_{k-1}{ }^{\lambda}\left(\bar{x}^{\lambda}\left(t_{k-1}^{-}\right)\right)\right] \\
-\int_{t_{k-1}}^{t_{k}} T_{q}\left(t_{k}-s\right) \bar{f}^{\lambda}\left(s, \bar{x}^{\lambda}(s),\left(H \bar{x}^{\lambda}\right)(s)\right) d s \\
-\Psi_{k-1,1}^{t_{k}} \mathcal{R}\left(\lambda, \Psi_{k-1,1}^{t_{k}}\right)\left\{x_{k}-S_{q}\left(t_{k}-t_{k-1}\right)\left[\bar{x}^{\lambda}\left(t_{k-1}^{-}\right)+\bar{I}_{k-1}^{\lambda}\left(\bar{x}^{\lambda}\left(t_{k-1}^{-}\right)\right)\right]\right. \\
\left.-\int_{t_{k-1}}^{t_{k}} T_{q}\left(t_{k}-s\right) \bar{f}^{\lambda}\left(s, \bar{x}^{\lambda}(s),\left(H \bar{x}^{\lambda}\right)(s)\right) d s\right\}, \quad k=2, \ldots, m+1 .
\end{gathered}
$$

From (2.3) we have $I-\Psi_{t_{k-1}, i}^{t_{k}} \mathcal{R}\left(\lambda, \Psi_{t_{k-1}, i}^{t_{k}}\right)=\lambda \mathcal{R}\left(\lambda, \Psi_{t_{k-1}, i}^{t_{k}}\right), i=1,2$, and

$$
x_{t_{1}}-\bar{x}^{\lambda}\left(t_{1}\right)=\lambda \mathcal{R}\left(\lambda, \Psi_{0,1}^{t_{1}}\right)\left\{x_{1}-S_{q}\left(t_{1}\right)\left(x_{0}-\bar{g}^{\lambda}\left(\bar{x}^{\lambda}\right)\right)\right.
$$




$$
\begin{aligned}
& \left.-\int_{0}^{t_{1}} T_{q}\left(t_{1}-s\right) \bar{f}^{\lambda}\left(s, \bar{x}^{\lambda}(s),\left(H \bar{x}^{\lambda}\right)(s)\right) d s\right\} \\
& x_{t_{k}}-\bar{x}^{\lambda}\left(t_{k}\right)=\lambda\left[\mathcal{R}\left(\lambda, \Psi_{k-1,1}^{t_{k}}\right)+\mathcal{R}\left(\lambda, \Psi_{k-1,2}^{t_{k}}\right)\right] \\
& \times\left\{x_{k}-S_{q}\left(t_{k}-t_{k-1}\right)\left[\bar{x} \lambda\left(t_{k-1}^{-}\right)+{\overline{I_{k-1}}}^{\lambda}\left(\bar{x}^{\lambda}\left(t_{k-1}^{-}\right)\right)\right]\right. \\
& \left.\left.-\int_{t_{k-1}}^{t_{k}} T_{q}\left(t_{k}-s\right) \bar{f}^{\lambda}\left(s, \bar{x}^{\lambda}(s),\left(H \bar{x}^{\lambda}\right)(s)\right)\right] d s\right\}, k=2, \ldots, m+1 .
\end{aligned}
$$

Since compactness of both $S_{q}(t)_{t>0}$ and $T_{q}(t)_{t>0}$ hold, and also boundedness of $\bar{f}^{\lambda}, \bar{g}^{\lambda}$ and $\overline{I_{k-1}} \lambda$, we can use on (4.1)-(4.2) the fact that $\lambda \mathcal{R}\left(\lambda, \Psi_{t_{k-1}, i}^{t_{k}}\right) \rightarrow 0$ in the strong operator topology as $\lambda \rightarrow 0^{+}, i=1,2$. This gives $\left\|x_{t_{k}}-\bar{x} \lambda\left(t_{k}\right)\right\|_{\alpha} \rightarrow 0$ as $\lambda \rightarrow 0^{+}, i=1,2$. Hence, the impulsive non-local fractional control system (1.1) is approximately controllable on $t \in[0, b] \backslash\left\{t_{1}, \ldots, t_{m}\right\}$.

\section{Optimality}

Let $Y$ be a separable reflexive Banach space and $w_{f}(Y)$ represent a class of nonempty, closed and convex subsets of $Y$. The multifunction $w: I \longrightarrow w_{f}(Y)$ is measurable and $w(\cdot) \subset E$, where $E$ is a bounded set of $Y$. We give the admissible control set as follows:

$$
U_{a d}=\left\{(u, v) \in L^{1}(E) \times L^{1}(E) \mid u(t), v(t) \in w(t) \text { a.e. }\right\} \neq \varnothing .
$$

Consider the following impulsive nonlocal fractional control system:

$$
\left\{\begin{array}{l}
{ }^{C} D_{t}^{q} x(t)=A x(t)+f(t, x(t),(H x)(t))+\mathcal{B} u(t), t \in(0, b] \backslash\left\{t_{1}, t_{2}, \ldots, t_{m}\right\}, \\
x(0)+g(x)=x_{0} \in X, \\
\triangle x\left(t_{i}\right)=I_{i}\left(x\left(t_{i}^{-}\right)\right)+\mathcal{D} v\left(t_{i}^{-}\right), i=1,2, \ldots, m, \quad(u, v) \in U_{a d},
\end{array}\right.
$$

where $\mathscr{B}, \mathscr{D} \in L^{\infty}(I, L(Y, X))$. It is clear that $\mathcal{B} u, \mathscr{D} v \in L^{1}(I, X)$ for all $(u, v) \in$ $U_{a d}$. Let $x^{u, v}$ be a mild solution of system (5.1) corresponding to controls $(u, v) \in$ $U_{a d}$. We consider the Bolza problem $(B P)$ : find an optimal triplet $\left(x^{0}, u^{0}, v^{0}\right) \in$ $P C(I, X) \times U_{a d}$ such that $\mathcal{I}\left(x^{0}, u^{0}, v^{0}\right) \leq \mathcal{L}\left(x^{u, v}, u, v\right)$, for all $(u, v) \in U_{a d}$, where

$$
\mathcal{L}\left(x^{u, v}, u, v\right)=\sum_{i=1}^{m+1}\left[\Phi\left(x^{u, v}\left(t_{i}\right)\right)+\int_{t_{i-1}}^{t_{i}} \mathscr{L}\left(t, x^{u, v}(t), u(t), v(t)\right) d t\right],
$$

$i=1, \ldots, m+1$. The following extra assumptions are needed:

$\left(\mathrm{H}_{6}\right)$ The functional $\mathscr{L}: I \times X \times Y^{2} \rightarrow \mathbb{R} \cup\{\infty\}$ is Borel measurable.

$\left(\mathrm{H}_{7}\right) \mathscr{L}(t, \cdot, \cdot, \cdot)$ is sequentially lower semi-continuous on $X \times Y^{2}$, a.e. on $I$.

$\left(\mathrm{H}_{8}\right) \mathscr{L}(t, \cdot, \cdot)$ is convex on $Y^{2}$ for each $x \in X$ and almost all $t \in I$. 
$\left(\mathrm{H}_{9}\right)$ There is a non-negative function $\varphi \in L^{\infty}(I, \mathbb{R})$ and $c_{1}, c_{2}, c_{3} \geq 0$ such that $\mathscr{L}(t, x, u, v) \geq \varphi(t)+c_{1}\|x\|+c_{2}\|u\|_{Y}^{p}+c_{3}\|v\|_{Y}^{p}$.

$\left(\mathrm{H}_{10}\right)$ The functional $\Phi: X \rightarrow \mathbb{R}$ is continuous and non-negative.

Theorem 3. If $\left(H_{6}\right)-\left(H_{10}\right)$ hold together with the assumptions of Theorem 1, then the Bolza problem $(B P)$ admits at least one optimal triplet on $P C \times U_{a d}$.

Proof. Assume $\inf \left\{g\left(x^{u, v}, u, v\right) \mid(u, v) \in U_{a d}\right\}=\delta<+\infty$. From $\left(\mathrm{H}_{6}\right)-\left(\mathrm{H}_{10}\right)$,

$$
\begin{aligned}
& \mathcal{J}\left(x^{u, v}, u, v\right) \\
& \quad \geq \sum_{i=1}^{m+1}\left[\Phi\left(x^{u, v}\left(t_{i}\right)\right)+\int_{t_{i-1}}^{t_{i}}\left\{\varphi(t)+c_{1}\|x(t)\|+c_{2}\|u(t)\|_{Y}^{p}+c_{3}\|v(t)\|_{Y}^{p}\right\} d t\right] \\
& \geq \geq-\eta>-\infty, i=1, \ldots, m+1 .
\end{aligned}
$$

Here, $\eta$ is a positive constant, i.e., $\delta \geq-\eta>-\infty$. By the definition of infimum, there exists a minimizing sequence of feasible triplets $\left\{\left(x^{n}, u^{n}, v^{n}\right)\right\} \subset \mathcal{A}_{a d}$, where $\mathcal{A}_{a d} \equiv\left\{(x, u, v) \mid x\right.$ is a mild solution of system (5.1) corresponding to $\left.(u, v) \in U_{a d}\right\}$, such that $\mathcal{f}\left(x^{n}, u^{n}, v^{n}\right) \rightarrow \delta$ as $m \rightarrow+\infty$. As $\left\{\left(u^{n}, v^{n}\right)\right\} \subseteq U_{a d}$ and $\left\{u^{n}, v^{n}\right\}$ is bounded in $L^{1}(I, Y)$, then there exists a subsequence, still denoted by $\left\{\left(u^{n}, v^{n}\right)\right\}$, and $u^{0}, v^{0} \in L^{1}(I, Y)$, such that $\left(u^{n}, v^{n}\right) \stackrel{\text { weakly }}{\longrightarrow}\left(u^{0}, v^{0}\right)$ in $L^{1}(I, Y) \times L^{1}(I, Y)$. Since the admissible control set $U_{a d}$ is convex and closed, by Marzur lemma, we have $\left(u^{0}, v^{0}\right) \in U_{a d}$. Suppose that $x^{n}$ is a mild solution of system (5.1), corresponding to $u^{n}$ and $v^{n}$, that satisfies

$$
x^{n}(t)=S_{q}(t)\left(x_{0}-g\left(x^{n}\right)\right)+\int_{0}^{t} T_{q}(t-s)\left(f\left(s, x^{n}(s),\left(H x^{n}\right)(s)\right)+\mathscr{B} u^{n}(s)\right) d s,
$$

for $t \in\left[0, t_{1}\right]$, and

$$
\begin{aligned}
x^{n}(t)=S_{q}\left(t-t_{i}\right)\left[x^{n}\left(t_{i}^{-}\right)\right. & \left.+I_{i}\left(x^{n}\left(t_{i}^{-}\right)\right)+\mathscr{D} v^{n}\left(t_{i}^{-}\right)\right] \\
& +\int_{t_{i}}^{t} T_{q}(t-s)\left[f\left(s, x^{n}(s),\left(H x^{n}\right)(s)\right)+\mathscr{B} u^{n}(s)\right] d s
\end{aligned}
$$

for $t \in\left(t_{i}, t_{i+1}\right], i=1, \ldots, m$. From $\left(\mathrm{H}_{2}\right)$, the non-linear function $f$ is bounded and continuous. Then, there exists a subsequence (with the same notation) $\left\{f\left(s, x^{n},\left(H x^{n}\right)(s)\right)\right\}$ and $f\left(s, x^{0},\left(H x^{0}\right)(s)\right) \in L^{1}(I, X)$ such that $f\left(s, x^{n},\left(H x^{n}\right)(s)\right)$ converges weakly to $f\left(s, x^{0},\left(H x^{0}\right)(s)\right)$. Also, the same arguments on $\left(\mathrm{H}_{3}\right)$ and $\left(\mathrm{H}_{5}\right)$ yield other weak convergences of $g\left(x^{n}\right)$ and $I_{i}\left(x^{n}\right)$ to $g\left(x^{0}\right)$ and $I_{i}\left(x^{0}\right)$, respectively. Let us denote

$$
\begin{gathered}
\left(P_{1} x\right)(t)=S_{q}(t) g(x)+\int_{0}^{t} T_{q}(t-s)(f(s, x(s),(H x)(s))+\mathscr{B} u(s)) d s, \quad t \in\left[0, t_{1}\right], \\
\left(P_{2} x\right)(t)=S_{q}\left(t-t_{i}\right)\left[x\left(t_{i}^{-}\right)+I_{i}\left(x\left(t_{i}^{-}\right)\right)+\mathscr{D} v\left(t_{i}^{-}\right)\right]
\end{gathered}
$$




$$
+\int_{t_{i}}^{t} T_{q}(t-s)[f(s, x(s),(H x)(s))+\mathscr{B} u(s)] d s, t \in\left(t_{i}, t_{i+1}\right], i=1, \ldots, m .
$$

Obviously, $\left(P_{1} x\right)(t)$ and $\left(P_{2} x\right)(t)$ are strongly continuous operators. Thus, $\left(P_{1} x^{n}\right)(t)$ and $\left(P_{2} x^{n}\right)(t)$ strongly converge to $\left(P_{1} x\right)(t)$ and $\left(P_{2} x\right)(t)$, respectively. Next, we consider the system

$$
x^{0}(t)=S_{q}(t)\left(x_{0}-g\left(x^{0}\right)\right)+\int_{0}^{t} T_{q}(t-s)\left(f\left(s, x^{0}(s),\left(H x^{0}\right)(s)\right)+\mathscr{B} u^{0}(s)\right) d s,
$$

$t \in\left[0, t_{1}\right]$, and

$$
\begin{aligned}
x^{0}(t)=S_{q}\left(t-t_{i}\right)\left[x^{0}\left(t_{i}^{-}\right)\right. & \left.+I_{i}\left(x^{0}\left(t_{i}^{-}\right)\right)+\mathscr{D} v^{0}\left(t_{i}^{-}\right)\right] \\
& +\int_{t_{i}}^{t} T_{q}(t-s)\left[f\left(s, x^{0}(s),\left(H x^{0}\right)(s)\right)+\mathscr{B} u^{0}(s)\right] d s,
\end{aligned}
$$

$t \in\left(t_{i}, t_{i+1}\right], i=1, \ldots, m$. It is not difficult to check that $\left\|x^{n}(t)-x^{0}(t)\right\| \rightarrow 0$ as $n \rightarrow \infty$. Therefore, we can infer that $x^{n}$ strongly converges to $x^{0}$ in $P C(I, X)$ as $n \rightarrow \infty$. From assumptions $\left(\mathrm{H}_{6}\right)-\left(\mathrm{H}_{10}\right)$ and Balder's theorem, we get

$$
\begin{aligned}
\eta & =\lim _{n \rightarrow \infty} \sum_{i=1}^{m+1}\left[\Phi\left(x^{n}\left(t_{i}\right)\right)+\int_{t_{i-1}}^{t_{i}} \mathscr{L}\left(t, x^{n}(t), u^{n}(t), v^{n}(t)\right) d t\right] \\
& \geq \sum_{i=1}^{m+1}\left[\Phi\left(x^{0}\left(t_{i}\right)\right)+\int_{t_{i-1}}^{t_{i}} \mathscr{L}\left(t, x^{0}(t), u^{0}(t), v^{0}(t)\right) d t\right] \\
& =\mathscr{g}\left(x^{0}, u^{0}, v^{0}\right) \geq \eta, i=1, \ldots, m+1,
\end{aligned}
$$

which implies that $\mathcal{g}$ attains its minimum at $\left(x^{0}, u^{0}, v^{0}\right) \in P C(I, X) \times U_{a d}$.

\section{ACKNOWLEDGEMENT}

This research is part of first author's PhD project. Debbouche and Torres are supported by FCT and CIDMA through project UID/MAT/04106/2013.

\section{REFERENCES}

[1] Q. Chen, A. Debbouche, Z. Luo, and J. Wang, "Impulsive fractional differential equations with Riemann-Liouville derivative and iterative learning control," Chaos Solitons Fractals, vol. 102, pp. 111-118, 2017, doi: 10.1016/j.chaos.2017.03.024.

[2] N. D. Cong and H. T. Tuan, "Generation of nonlocal fractional dynamical systems by fractional differential equations," J. Integral Equations Appl., vol. 29, no. 4, pp. 585-608, 2017, doi: 10.1216/JIE-2017-29-4-585.

[3] A. Debbouche and V. Antonov, "Approximate controllability of semilinear Hilfer fractional differential inclusions with impulsive control inclusion conditions in Banach spaces," Chaos Solitons Fractals, vol. 102, pp. 140-148, 2017, doi: 10.1016/j.chaos.2017.03.023. 
[4] A. Debbouche and J. J. Nieto, "Sobolev type fractional abstract evolution equations with nonlocal conditions and optimal multi-controls," Appl. Math. Comput., vol. 245, pp. 74-85, 2014, doi: 10.1016/j.amc.2014.07.073.

[5] A. Debbouche, J. J. Nieto, and D. F. M. Torres, "Optimal solutions to relaxation in multiple control problems of Sobolev type with nonlocal nonlinear fractional differential equations," J. Optim. Theory Appl., vol. 174, no. 1, pp. 7-31, 2017, doi: 10.1007/s10957-015-0743-7.

[6] A. Debbouche and D. F. M. Torres, "Approximate controllability of fractional delay dynamic inclusions with nonlocal control conditions," Appl. Math. Comput., vol. 243, pp. 161-175, 2014, doi: 10.1016/j.amc.2014.05.087.

[7] A. Debbouche and D. F. M. Torres, "Sobolev type fractional dynamic equations and optimal multiintegral controls with fractional nonlocal conditions," Fract. Calc. Appl. Anal., vol. 18, no. 1, pp. 95-121, 2015, doi: 10.1515/fca-2015-0007.

[8] H. Gou and B. Li, "Local and global existence of mild solution to impulsive fractional semilinear integro-differential equation with noncompact semigroup," Commun. Nonlinear Sci. Numer. Simul., vol. 42, pp. 204-214, 2017, doi: 10.1016/j.cnsns.2016.05.021.

[9] A. A. Kilbas, H. M. Srivastava, and J. J. Trujillo, Theory and applications of fractional differential equations. Elsevier Science B.V., Amsterdam, 2006.

[10] S. Liu, A. Debbouche, and J. Wang, "On the iterative learning control for stochastic impulsive differential equations with randomly varying trial lengths," J. Comput. Appl. Math., vol. 312, pp. 47-57, 2017, doi: 10.1016/j.cam.2015.10.028.

[11] S. Liu, J. Wang, and Y. Zhou, "Optimal control of noninstantaneous impulsive differential equations," J. Franklin Inst., vol. 354, no. 17, pp. 7668-7698, 2017, doi: 10.1016/j.jfranklin.2017.09.010.

[12] A. Pazy, Semigroups of linear operators and applications to partial differential equations. Springer-Verlag, New York, 1983. doi: 10.1007/978-1-4612-5561-1.

[13] H. Qin, X. Zuo, J. Liu, and L. Liu, "Approximate controllability and optimal controls of fractional dynamical systems of order $1<q<2$ in Banach spaces," Adv. Difference Equ., pp. 2015:73, 17, 2015, doi: 10.1186/s13662-015-0399-5.

[14] B. Radhakrishnan and K. Balachandran, "Controllability of neutral evolution integrodifferential systems with state dependent delay,” J. Optim. Theory Appl., vol. 153, no. 1, pp. 85-97, 2012, doi: 10.1007/s10957-011-9934-z.

[15] R. Sakthivel, R. Ganesh, Y. Ren, and S. M. Anthoni, "Approximate controllability of nonlinear fractional dynamical systems," Commun. Nonlinear Sci. Numer. Simul., vol. 18, no. 12, pp. 3498 3508, 2013, doi: 10.1016/j.cnsns.2013.05.015.

[16] X.-B. Shu and Q. Wang, "The existence and uniqueness of mild solutions for fractional differential equations with nonlocal conditions of order $1<\alpha<2$," Comput. Math. Appl., vol. 64, no. 6, pp. 2100-2110, 2012, doi: 10.1016/j.camwa.2012.04.006.

[17] R. Triggiani, "A note on the lack of exact controllability for mild solutions in Banach spaces," SIAM J. Control Optimization, vol. 15, no. 3, pp. 407-411, 1977, doi: 10.1137/0315028. 
Authors' addresses

\section{Sarra Guechi}

Guelma University, Department of Mathematics, 24000 Guelma, Algeria

E-mail address: guechi.sara@yahoo.fr

Amar Debbouche

Guelma University, Department of Mathematics, 24000 Guelma, Algeria

E-mail address: amar_debbouchedyahoo.fr

Delfim F. M. Torres

University of Aveiro, DMat, CIDMA, 3810-193 Aveiro, Portugal

E-mail address: delfimeua.pt 